\title{
Hydrodynamics of formation of a microdispersed spray by the cup rotary atomizer
}

\author{
Ivan Sidorenko ${ }^{1 *}$, Peter Dogoda ${ }^{1}$, and Alexander Mashkov ${ }^{1}$ \\ ${ }^{1}$ V. I. Vernadsky Crimean Federal University, Academy of bioresources and nature management, \\ 295492, Agrarnoe urban settlement, Republic Of Crimea, Russian Federation
}

\begin{abstract}
This article is devoted to the study of the movement of a single drop inside an air-liquid jet formed by a cup atomizer. The relevance of the research topic is proved. The problem, which has been identified, is about using modern fan sprayers for chemical protection of agricultural crops, in particular, orchards and vineyards. As for mechanical spraying, the process of movement of the droplet inside the torch, the trajectory of droplet flight, as well as the dependence of these indicators on the parameters of the rotary atomizers are currently very little studied. Therefore, this article considers the laws describing the movement of one droplet on the surface of the rotating working element and one droplet flight in atmospheric air after escape from the surface of the rotary atomizer's cup. Research results are presented as the equations describing the dependence of the torch boundaries on the parameters of the rotary cup atomizer.
\end{abstract}

\section{Introduction}

Horticulture and viticulture occupy a significant place in modern agriculture of the Republic of Crimea and southern Russia. In these industries, according to many years of experience in fruit crops cultivation, the yield and quality of products depend to a large extent on the level of plant protection against pests and diseases. Efficiency of chemical crops treatment is determined by the following indicators: diameter of operating fluid droplets, density of protected surface coating, uniformity distribution of pesticide solution by tree crown or grape bush. Many years of experience in mechanization of chemical plants protection has shown that the optimal values above these indicators are provided by the implementation of low and ultra-low volume spraying. The essence of this method is the application of a pesticide solution in the form of fine drops on the surface under treatment. Their diameter varies within $d_{m}=1 \ldots 150 \mu \mathrm{m}$ [1]. Many years of experience in operation and experimental studies showed that with this size of droplets distribution of working fluid on the treated surface is uniform, the area and density of plant coating increase. However, the correct organization of drip flow flight is necessary to ensure that these requirements are fulfilled. Based on the identified problem, the purpose and objectives of the research were formulated.

\footnotetext{
* Corresponding author: vanya.sidorenko.84@mail.ru
} 
The purpose is to establish the dependence of the geometric characteristics of the drip flow on the design parameters of the rotary cup atomizer.

In order to achieve this goal, the following tasks must be solved:

- analyze existing data in the field of rotary atomizers theory;

- study the physical nature of the droplet formation process and motion of liquid particles on a rotating spherical surface;

- determine the forces influencing the drop before and after separation from the edge of the rotating surface;

- study the effect of the cup-shaped working element shape of the atomizer on the direction of the liquid particle movement.

\section{Materials and Methods}

Studies of a drip flow emitted from a rotary cup atomizer are based on classical laws of kinematics and material point dynamics under different kinds of motion. The shape of the fluid particle trajectory before and after separation from the edge of the spraying cup element is described using the basic concepts and statutes of geometry and trigonometry.

\section{Results and Discussion}

When studying the dynamical of mechanical systems with rotating elements focused on various operations (mixing, grinding, drying, balancing, spraying, etc.) of different substances, the problem arises about the interaction of moving elements (droplets) and these rotating elements (spraying cup) [2]. First of all, we should consider the process of disintegrating the working fluid into small droplets during the operation of a rotary atomizer. It includes several stages. The first stage involves the flow of working fluid on the pressure line inside the atomizer. In it, the agrochemical solution enters through a hollow shaft into the housing. Next, the working fluid passes through the dissector. It is a cylinder with small diameter round holes located on the periphery. In the second step, the working fluid, passing through the holes of the dissector, arrives as thin threads on the inner surface of the rapidly rotating cup. After that comes the third stage of the process. It provides for the disintegration of the pesticide solution into small droplets under the influence of centrifugal force. Analysis of existing data showed that this spraying method provides uniform size particles of working solution, diameter of which can be adjusted [3].

Based on the description of the atomizer operation process, the drop is formed when the liquid thread enters the rapidly rotating cup and begins to move on its surface. In this case, it can be concluded that the initial acceleration of the fluid particle is very small. Due to this, the drop moves on the surface of the rotary cup along an arcuate line. This phenomenon is typical of the non-inertial reference system. In it, the drop rotates with the cup. From classical mechanics it is known that the Coriolis force is perpendicular to the rotation axis and velocity of the particle, and in magnitude equals to[4]:

$$
\begin{aligned}
F_{K} & =2 \cdot m \cdot v_{R} \cdot \omega_{\text {Cup }} \cdot \sin \alpha, \\
F_{K} & =2 \cdot m \cdot \overrightarrow{v_{R}} \cdot \overrightarrow{\omega_{C u p}},
\end{aligned}
$$

where $\omega_{\text {Cup }}$ is the angular velocity of the rotating reference system;

$\alpha$-is the angle between the relative velocity vectors $v_{R}$ and $\omega_{C u p}$.

It is the Coriolis force that gives the trajectory of drop motion a spiral shape. 
Taking into account the laws of classical mechanics and dynamics of the material point, the scheme of forces acting on the drop at the moment of its separation from the edge of the rotary atomizer cup was formed (Fig. 1).

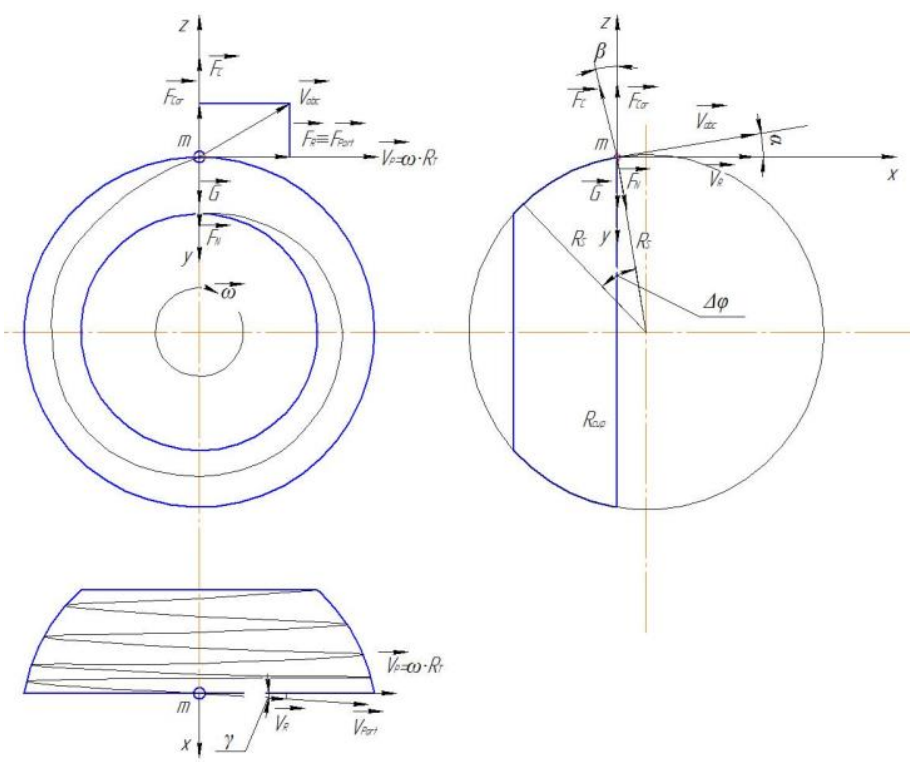

Fig. 1. Scheme of forces acting on the drop at the moment of its separation from the edge of the rotary atomizer cup.

Let us consider a liquid particle located at the top point of the cup edge circumference. At the same time, the force of gravity $G$ acts on the drop of mass $m$. Its vector is directed vertically downwards. At the moment of separation from the cup edge, the drop acquires linear velocity, which is also called circumferential [5]:

$$
v_{C}=\omega_{C u p} \cdot R_{C u p}
$$

where $R_{C u p}$ is the radius of the cup edge associated with the radius of the sphere $R_{S}$, forming the profile of the inner surface of the working element with ratio:

$$
R_{\text {Cup }}=R_{S} \cdot \cos \beta \text {. }
$$

Hence, the surface reaction force of the atomizer working element by the expression:

$$
F_{N}=\frac{G}{\cos \beta}=\frac{m \cdot g}{\cos \beta} .
$$

Rotational motion of the drop on the surface of the rotation cup around the atomizer axis is provided by centrifugal force [6]:

$$
F_{C}=\frac{\pi \cdot d_{m}^{3}}{6} \cdot \rho_{L} \cdot g .
$$


According to the force scheme, in the projection on the $Z$ axis, the equations above the specified forces acting on the drop will be as follows:

$$
\begin{aligned}
& F_{N}=G=m \cdot g . \\
& F_{C}=\frac{\pi \cdot d_{m}^{3}}{6} \cdot \rho_{L} \cdot g \cdot \cos \beta .
\end{aligned}
$$

From the scheme of forces we see:

$$
\frac{\pi \cdot d_{m}^{3}}{6} \cdot \rho_{L} \cdot g \cdot \cos \beta=m \cdot g
$$

The Coriolis force $F_{C o r}$ is always directed from the horizontal atomizer axis perpendicular to it, remaining to act in the plane of rotation. According to the material point dynamics laws, the relative velocity $v_{R}$, as the angular $\omega_{C o r}$, is perpendicular to the plane of spinning. Consequently, they both lie in the same plane. So the Coriolis force $F_{C O R}$ is directed perpendicular to them. Together with the relative velocity $v_{C o r}$ they define the slope of the absolute velocity vector $v_{a b s}$ to the horizon.

Coriolis acceleration is defined by the formula [4]:

$$
\overrightarrow{a_{C o r}}=2 \cdot\left[v_{C o r} \cdot \omega\right]
$$

Based on the forces scheme, the absolute velocity is determined by the ratio:

$$
v_{a b s}=\frac{v_{R}}{\cos \alpha}
$$

By combining the equations (10) and (11) into the system, we will get:

$$
\left\{\begin{array}{l}
a_{C o r}=2 \cdot v_{R} \cdot \omega \\
v_{a b s}=\frac{v_{R}}{\cos \alpha}
\end{array}\right.
$$

From the second equation of the system (12), we express $v_{R}$ and add the resulting ratio into the first:

$$
a_{C o r}=2 \cdot v_{a b s} \cdot \omega_{c u p} \cdot \cos \alpha
$$

We project centrifugal $F_{C}$ and Coriolis $F_{C o r}$ forces onto the Zaxis:

$$
F_{C o r}=F_{C} \cdot \cos \beta=\frac{\pi \cdot d_{m}^{3}}{6} \cdot \rho_{L} \cdot g \cdot \cos \beta
$$

Let us set in the ratio (14) the formula for determining the Coriolis force: 


$$
2 \cdot m \cdot v_{R} \cdot \omega=\frac{\pi \cdot d_{m}^{3}}{6} \cdot \rho_{L} \cdot g \cdot \cos \beta
$$

In this case, the expression to determine the relative velocity $v_{R}$ is as follows:

$$
v_{R}=\frac{\pi \cdot d_{m}^{3} \cdot \rho_{L} \cdot g \cdot \cos \beta}{12 \cdot \omega_{\text {Cup }} \cdot m}
$$

where $m$ is the mass of the drop, $\mathrm{kg}$.

From the course of classical mechanics it is known:

$$
\omega=\frac{\pi \cdot n_{\text {Cup }}}{30}
$$

Taking into account the expression of angular velocity, we will make some transformations in the formula (17):

$$
v_{R}=\frac{2,5 \cdot d_{m}^{3} \cdot \rho_{L} \cdot g \cdot \cos \beta}{n_{\text {Cup }} \cdot m} .
$$

Knowing $v_{R}$, we bring out a formula to determine the drop motion acceleration relative to the surface of the rotating cup of the spray during the time $\Delta t$ :

$$
a_{R}=\frac{v_{R}}{\Delta t}=\frac{2,5 \cdot d_{m}^{3} \cdot \rho_{L} \cdot g \cdot \cos \beta}{\Delta t \cdot n_{\text {Cup }} \cdot m} .
$$

Taking into account the formula (19), one can derive the equation of the force of the relative $F_{R}$ motion of the drop:

$$
F_{R}=m \cdot a_{R}=\frac{2,5 \cdot d_{m}^{3} \cdot \rho_{L} \cdot g \cdot \cos \beta}{\Delta t \cdot n_{\text {Cup }}} .
$$

Since the drop moves on the surface of the rotating cup in a non-inertial reference system, the shift from the radial direction is provided by the Coriolis force $F_{C o r}$ and relative motion $F_{R}$. Therefore, the direction vectora of the resulting (absolute) force $F$ acting on the drop is determined by the angle $\alpha$ between Coriolis force $F_{C o r}$ and relative motion $F_{R}$ :

$$
\operatorname{tg} \alpha=\frac{F_{C o r}}{F_{R}}=\frac{\pi \cdot d_{m}^{3} \cdot \rho_{L} \cdot g \cdot \cos \beta}{6} \cdot \frac{\Delta t \cdot n_{\text {Cup }}}{2,5 \cdot d_{m}^{3} \cdot \rho_{L} \cdot g \cdot \cos \beta}=\frac{\Delta t \cdot n_{C u p}}{15} .
$$

From the forces scheme it can be seen that the absolute velocity vector is an extension of the drop motion line on the inner surface of the cup, representing a loxodrome 
- a curve passing by spherical surface of rotation, crossing all meridians at a constant angle $\gamma$ [7]. Vector of the circumferential (linear) velocity, $v_{C}$ is directed along the meridian. With it, the relative velocity vector $v_{R}$ lies in the same plane. Hence, the loxonometric path angle $\gamma$ is the angle between the vectors $v_{C}$ and $v_{R}$ :

$$
\cos \gamma=\frac{\overrightarrow{v_{C}}}{\overrightarrow{v_{R}}} .
$$

Since the loxodrome crosses all meridians at the same angle $\gamma$, the profile arc of the inner cup surface can be divided into equal parts:

$$
L_{1}=L_{2}=\ldots=L_{n}=\frac{\pi \cdot R_{S}}{2} \cdot \operatorname{tg} \gamma
$$

The length of the line $S$ movement of the point on the rotating sphere surface is calculated according to the properties of the loxodroma by the formula:

$$
\mathrm{S}=\frac{R_{S}-R_{\text {Cup }}}{\cos \gamma}=\frac{\left(R_{S}-R_{\text {Cup }}\right) \cdot v_{R}}{v_{C}} .
$$

The line of spherical motion of a drop can also be thought of as a special case of an Archimedean spiral with increment $S=k \cdot \varphi$ ( $k$ - the displacement of the material point (drop) on the ray) [8].

The latter can be thought of as a chord, length of which is determined using the ratios:

$$
\Delta \varphi=\frac{L}{D}=\frac{L}{2 R_{S}}
$$

where $L$ is the arc length, $\mathrm{m}$;

$R_{S}$ - radius of cup profile sphere, m;

$\Delta \varphi$ is the rotation angle of the radius $R_{S}$ of the cup when a drop passes the distance equal to the length of the arc $L$ (central angle).

Hence:

$$
\Delta \varphi=\frac{\left(R_{S}-R_{C u p}\right) \cdot v_{R}}{2 \cdot R_{S} \cdot v_{C}}
$$

From the course of classical mechanics it is known that the angular velocity $\omega$ is determined by the ratio of the displacement angle of $\Delta \varphi$ radius of the circumference arc on time of its rotation $\Delta t \omega=\Delta \varphi / \Delta t[9]$.

Thus, taking into account the expression to determine the displacement angle $\Delta \varphi$ of the radius $R_{S}$ of the sphere profile of the cup into the equation (21), as well as taking into account the ratios (17) and (26), making some mathematical transformations, we will get: 


$$
\operatorname{tg} \alpha=\frac{4500 \cdot\left(R_{S}-R_{\text {Cup }}\right) \cdot g}{\pi^{2} \cdot R_{S}^{2} \cdot n_{\text {Cup }}^{2}} .
$$

The equation (27) reflects the dependence of the angle $\alpha$ between the vectors of relative $v_{R}$ and absolute $v_{a b s}$ velocity of the drop, separated from the edge of the rotary atomizer cup. This indicator characterizes the taper rate of the fine-dispersed torch.

\section{Conclusion}

The results of the research presented in the article allowed to establish the dependence between the design and mode parameters of the rotary atomizer (radius of the edge circumference $R_{\text {Cup }}$ and radius of the cup sphere profile $R_{S}$ ) and the geometric characteristic of the fine-drop torch - the taper angle $\alpha$. The formula reflecting this relationship is derived taking into account the forces acting on the drop before and after separation from the rotary cup-shaped working element. Its composition includes only the characteristics of the atomizer, which can be determined in the production conditions of agricultural enterprises. Therefore, this formula can be used in the design and creation of rotary cup atomizers for low and ultra-low volume sprayers.

\section{Reference}

1. V.F. Dunsky, N.V. Nikitin, M.S. Sokolov Pesticide aerosols, (M.: Nauka, 1982)

2. E.S. Shalimova, Herald Of the Moscow Univ. Math. Mechanics, 4, 43-50 (2014)

3. I.D. Sidorenko, Development of rotary atomizer design for ultra-low volume fan sprayer, II Interdisciplinary Scientific-Practical conference of young scientists on promising directions of modern science development "Akademik Vernadsky" within the framework of the festival "DAYS OF SCIENCE of CFU named after. V.I. VERNADSKY" Collection of abstracts of participants, 107-110 (2016)

4. I.V. Saveliev, General Physics course, Mechanics, oscillations and waves, molecular physics (Publishing House "Nauka". Main editorial of physical-mathematical literature, M. 1970)

5. N.M. Rogachev, Physics course: Textbook. 2nd ed., str. (St.Petersburg: "Lan" Publishing House, 2010)

6. V.F. Dunsky, N.V. Nikitin, M.S. Sokolov, Monodisperse aerosols (M.: Nauka, 1975)

7. V.S. Mikhailov, V.G. Kudryavtsev, V.S. Davydov, Basic formulas of orthodromy. Methods of its assignment, Navigation and pilot (Kyiv, 2009)

8. A.I. Kurnosenko, Notes of scientific seminars, PDMI, 353, 93-115 (2009)

9. T.I. Trofimova, Course of physics: textbook for universities. 11th ed., (Publishing Center “Academy”, 560 Moscow, 2006) 